\title{
Fosfomycin: Mechanism and Resistance
}

\author{
Lynn L. Silver \\ LL Silver Consulting, LLC, Springfield, New Jersey 07081 \\ Correspondence: silverly@comcast.net
}

Fosfomycin, a natural product antibiotic, has been in use for $>20$ years in Spain, Germany, France, Japan, Brazil, and South Africa for urinary tract infections (UTIs) and other indications and was registered in the United States for the oral treatment of uncomplicated UTIs because of Enterococcus faecalis and Escherichia coli in 1996. It has a broad spectrum, is bactericidal, has very low toxicity, and acts as a time-dependent inhibitor of the MurA enzyme, which catalyzes the first committed step of peptidoglycan synthesis. Whereas resistance to fosfomycin arises rapidly in vitro through loss of active transport mechanisms, resistance is rarely seen during therapy of UTIs, seemingly because of the low fitness of the resistant organisms. Recently, interest has grown in the use of fosfomycin against multidrugresistant (MDR) pathogens in other indications, prompting the advent of development in the United States of a parenteral formulation for use, initially, in complicated UTIs. Whereas resistance has not been problematic in the uncomplicated UTI setting, it remains to be seen whether resistance remains at bay with expansion to other indications.

$\mathrm{F}_{\mathrm{c} i \sin }^{\mathrm{og}}$ osfomycin, originally called phosphonomycin, is a broad spectrum antibiotic first found in fermentation broths of Streptomyces fradiae (ATCC 21096) in Spain through a collaborative effort of Merck and the Compañía Española de Penicilina y Antibióticos (CEPA) (Hendlin et al. 1969). Fosfomycin was initially developed in Europe by CEPA and has been in use since the early 1970s, initially as an IV preparation of the disodium salt and later as an oral formulation of fosfomycin trometamol. Its primary use in Spain, Germany, France, Japan, Brazil, and South Africa has been as an oral treatment for urinary tract infections (UTIs) but it has also been used more broadly in other indications (Falagas et al. 2008, 2009, 2010a). Fosfomycin was approved for use in the United States (as
Monurol, fosfomycin tromethamine [same as trometamol]) in 1996 for treatment by singledose oral therapy of uncomplicated UTIs (acute cystitis) in women caused by Escherichia coli and Enterococcus faecalis. With the problem of increasing resistance to other antibiotics, parenteral use of fosfomycin has been studied in therapy of a variety of infections because it is active against many multidrug-resistant (MDR) pathogens (Falagas et al. 2009) and is now under development in the United States for parenteral treatment of complicated UTIs (Zavante 2016).

\section{DISCOVERY AND SPECTRUM}

Fosfomycin (Fig. 1A) is a phosphonic acid antibiotic discovered in Spain in a fermentation

Editors: Lynn L. Silver and Karen Bush

Additional Perspectives on Antibiotics and Antibiotic Resistance available at www.perspectivesinmedicine.org

Copyright (C) 2017 Cold Spring Harbor Laboratory Press; all rights reserved; doi: 10.1101/cshperspect.a025262 Cite this article as Cold Spring Harb Perspect Med 2017;7:a025262 
L.L. Silver

A

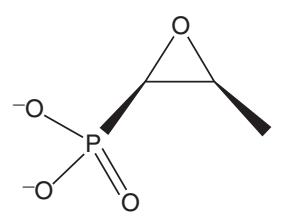

B

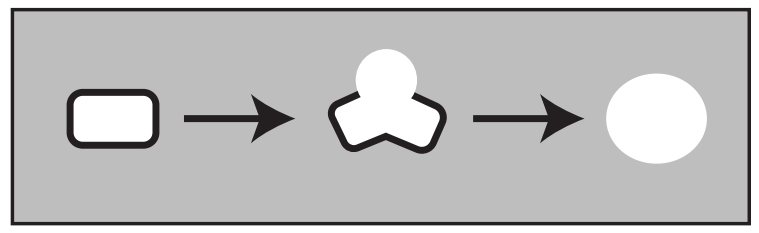

Figure 1. Fosfomycin and the screen in which it was discovered. (A) Structure of fosfomycin. (B) Schematic of SPHERO assay. Gram-negative rods grown in osmotically protective medium, when treated with inhibitors of peptidoglycan synthesis, will show morphological changes leading to the production of round cells called spheroplasts, which are highly differentiable microscopically from normal rods and debris. The assay was run in plastic trays with wells for growth of cells and the wells viewed, after incubation, with a stereomicroscope.

broth of $S$. fradiae by means of a Merck screen for inhibition of peptidoglycan synthesis, the SPHERO assay (Gadebusch et al. 1992). In this morphological assay, schematized in Figure 1B, Gram-negative bacilli are grown in osmotically protective medium and treated for several doublings with test samples. Inhibitors of steps in the synthesis of peptidoglycan will lead to the production of microscopically recognizable refractile spheroplasts. Fosfomycin is also produced by several other Streptomyces, including Streptomyces viridochromogenes (ATCC21240) and Streptomyces wedmorensis (ATCC 21239), as well as Pseudomonas syringae (Shoji et al. 1986), Pseudomonas viridiflava, and Pseudomonas fluorescens (Katayama et al. 1990).

Early tests indicated that fosfomycin was efficacious via IV dosing against intraperitoneal murine infections by specific isolates of E. coli, Klebsiella pneumoniae, Pseudomonas aeruginosa, Proteus vulgaris, Salmonella schottmuelleri, Staphylococcus aureus, and Streptococcus pyogenes-although $\mathrm{ED}_{50} \mathrm{~s}$ (median effective dose) against $K$. pneumoniae, $P$. aeruginosa, and $S$. pyogenes were high (although attainable and safe) (Hendlin et al. 1969). More recent reports note its broad spectrum, including activity against many important pathogens such as $S$. aureus (including methicillin-resistant $S$. aureus [MRSA]), Staphylococcus epidermidis, Strepto- coccus pneumoniae, E. faecalis, E. coli, Proteus species, K. pneumoniae, Enterobacter species, Serratia marcescens, and Salmonella typhi. Whereas $P$. aeruginosa shows variable susceptibility, fosfomycin has shown anti-pseudomonal efficacy especially in combinations with cefepime, aztreonam, and meropenem (Falagas et al. 2008). Acinetobacter, Vibrio fischeri, Chlamydia trachomatis, and Bacteroides species are resistant to fosfomycin (Falagas et al. 2008; Karageorgopoulos et al. 2012). Fosfomycin is not cross-resistant with other antibiotics because of its unique structure and mechanism of action.

\section{MECHANISM OF ACTION}

Fosfomycin is an inhibitor of the MurA enzyme, UDP- $N$-acetylglucosamine-enolpyruvyltransferase, that catalyzes the first committed step in peptidoglycan synthesis, the reaction of UDP-Nacetylglucosamine (UDP-GlcNAc) with phosphoenolpyruvate (PEP) to form UDP-GlcNAcenoylpyruvate plus inorganic phosphate (shown in Fig. 2). The finding that competition of fosfomycin inhibition by PEP implied that fosfomycin was acting as a PEP analog (Kahan et al. 1974). Fosfomycin covalently binds, in a timedependent reaction involving nucleophilic attack by the cysteine 115 residue of E. coli MurA on the epoxide of fosfomycin (Marquardt et al. 
1994), and C115 is considered responsible for the catalytic action on PEP in the synthetic reaction (Wanke and Amrhein 1993; Brown et al. 1994).

In vitro, the MurA reaction proceeds with UDP-GlcNAc binding to the so-called open form of MurA, leading to a structural change to a closed form to which PEP binds (Skarzynski et al. 1996) leading to a tetrahedral intermediate and product release (Eschenburg et al. 2005). Even though it acts as an analog of PEP, fosfomycin's interaction with MurA is highly selective (Kahan et al. 1974; Marquardt et al. 1994) and shows extremely low toxicity. Its $\mathrm{LD}_{50}$ (median lethal dose) in mice by intraperitoneal dosing of $\mathrm{Na}_{2}$-fosfomycin was $4 \mathrm{~g} / \mathrm{kg}$, lethality likely being because of sodium content (Hendlin et al. 1969).

The existence of the MurA analog of $\mathrm{Myco}$ bacterium tuberculosis that is naturally resistant to fosfomycin and has an asparagine in the position comparable to the $\mathrm{C} 115$ led to studies revealing further details of MurA reactions. Replacement of the C115 of the E. coli enzyme by asparagine (to yield a C115D enzyme) leads to fosfomycin resistance (Kim et al. 1996) and, as expected, replacement of asparagine in the M. tuberculosis enzyme with cysteine endows it with fosfomycin sensitivity (De Smet et al. 1999). This indicates that C115 is not necessarily required for the catalytic activity of the enzyme, but this activity can be supplied by the asparagine acting as a general acid (Kim et al. 1996). Substitution of C115 of Enterobacter cloacae MurA by serine (C115S) yielded an enzyme with the reaction products, UDP-GlcNAcenoylpyruvate and $\mathrm{Pi}$, bound in the active site
(Eschenburg et al. 2005), leading the authors to conclude that C115 is necessary for turnover and release of the products. They reasoned that, in C115S, other residues could carry out the catalytic step and that, in the C115D enzyme, the flexible asparagine should be able to catalyze the release of the reaction products.

Recent work (Zhu et al. 2012) indicates that, in the cell, the activity of MurA is subject to regulation by the binding of UDP-NAc-muramic acid (UDP-MurNAc), the product of the MurB enzyme, to MurA. In the presence of bound UDP-MurNAc, PEP covalently attaches to $\mathrm{C} 115$, leading to the formation of a "locked" dormant tertiary complex (MurA:PEP-UDPMurNAc), which is the predominant form of cellular MurA. This had apparently been missed previously because much crystallographic work with MurA had been done by diluting samples into phosphate buffer, which releases the ligands. Extensive crystallographic findings by these authors led them to a model for the regulatory and reaction schemes: when the UDPMurNAc to UDP-GlcNAc ratio in the cell decreases, UDP-GlcNAc replaces UDP-MurNAc in the dormant complex in a rapidly reversible manner, leading to formation of the tetrahedral reaction intermediate that then yields the products, still bound in the active site. PEP enters, reacts with $\mathrm{C} 115$, and displaces $\mathrm{Pi}$, stimulating the reversible exchange of UDP-GlcNAc with UDP-GlcNAc-enoylpyruvate, leading to its release to restart the cycle. The authors speculate that the existence of the locked dormant complex may explain why discovery of reversible, noncovalent inhibitors of MurA has been difficult. In this model, then, the covalent interac-

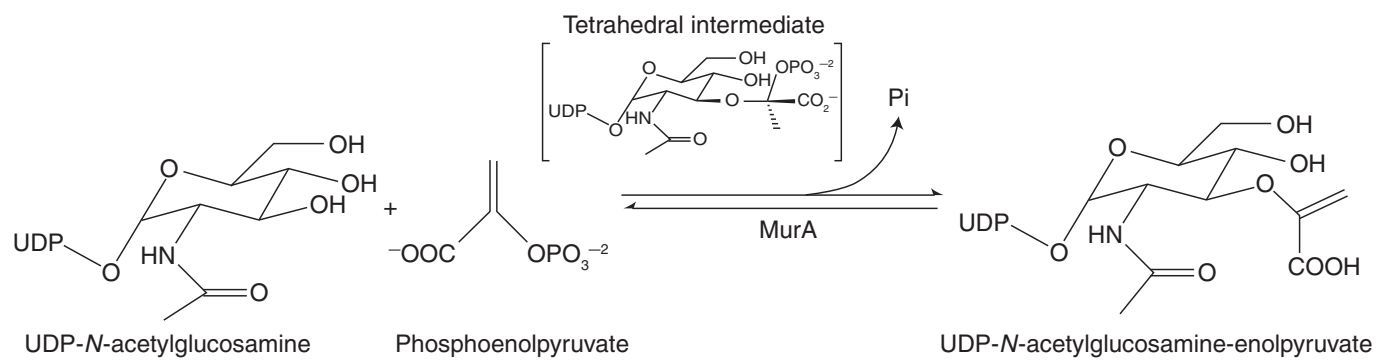

Figure 2. Overall reaction performed by the MurA enzyme. 
L.L. Silver

tion of PEP with C115 is necessary for formation of the dormant complex and also for release of the reaction products (as had been proposed by Eschenburg et al. 2005) but is not necessary for catalysis by the enzyme that may be supplied by other residues (as had been proposed by Kim et al. 1996).

In low GC Gram-positives, there are two "murA" genes, murA and mur $Z$, the products of which are structurally very similar (Du et al. 2000; Blake et al. 2009). In the case of the $S$. pneumoniae and $S$. aureus enzymes, each gene product is capable of sustaining peptidoglycan synthesis and both isozymes are sensitive to fosfomycin in vitro (Blake et al. 2009). Neither gene is essential but a double deletion is not viable. In Bacillus subtilis (Kobayashi et al. 2003; Kock et al. 2004) and Bacillus anthracis (Kedar et al. 2008), however, only the murA gene is essential and, evidently, the second enzyme cannot substitute.

\section{FOSFOMYCIN UPTAKE}

In E. coli, two carrier-dependent systems can actively transport fosfomycin. Initial work showed that fosfomycin could be transported by the system for uptake of $\alpha$-glycerophosphate and that mutants in the $g l p T$ gene, encoding the $\alpha$-glycerophosphate permease, were 30 -fold more resistant than the isogenic parent strain. Whereas $\alpha$-glycerophosphate will induce expression of GlpT, the basal levels of GlpT are sufficient for fosfomycin uptake (Kahan et al. 1974). The GlpT system is widespread, found at least in P. aeruginosa, E. coli, Salmonella, Shigella flexneri, Klebsiella, Haemophilus influenzae, S. aureus, B. subtilis, E. faecalis, and Rickettsia prowazekii (Kahan et al. 1974; Lemieux et al. 2004). It was also shown that the blood component, glucose-6-phosphate (G-6-P), could induce the hexose-phosphate uptake system, UhpT, to levels sufficient for fosfomycin transport (Zimmerman et al. 1969; Kahan et al. 1974). The UhpT system is limited to Enterobacteriaceae (with the exception of Proteus species) and S. aureus (Winkler 1973). Importantly, Pseudomonas does not have a UhpT system and so solely uses $g l p T$ for uptake (Winkler 1973; Castañeda-García et al. 2009).

\section{FOSFOMYCIN RESISTANCE}

The mechanisms of resistance to fosfomycin have been recently reviewed (Karageorgopoulos et al. 2012; Castañeda-García et al. 2013; Nikolaidis et al. 2014). It should be noted that the breakpoints for fosfomycin susceptibility have been formalized for few species and are different for the Clinical and Laboratory Standards Institute (CLSI) and the European Committee on Antimicrobial Susceptibility Testing (EUCAST). EUCAST break points for Enterobacteriaceae (oral or IV) and $S$. aureus (IV only) are $\mathrm{S} \leq$ $32 \mathrm{mg} / \mathrm{L}$ and $\mathrm{R}>32 \mathrm{mg} / \mathrm{L}$ (in the presence of $25 \mathrm{mg} / \mathrm{L}$ G-6-P). Wild-type isolates of Pseudomonas species minimum inhibitory concentrations (MICs) $\leq 128 \mathrm{mg} / \mathrm{L}$ have been treated with combinations (European Committee on Antimicrobial Susceptibility Testing 2016). CLSI breakpoints for E. coli and E. faecalis are $\mathrm{S} \leq 64 \mathrm{mg} / \mathrm{L}, \mathrm{I} 128 \mathrm{mg} / \mathrm{L}, \mathrm{R} \geq 256 \mathrm{mg} / \mathrm{L}$ (also in the presence of G-6-P) (Performance Standards for Antimicrobial Susceptibility Testing 2012).

\section{Mutational Resistance}

Resistance to fosfomycin arises in E. coli at high frequencies in vitro, because of loss of transport systems required for uptake. As noted above, in E. coli, uptake of fosfomycin can be mediated by the GlpT permease system, whose main substrate is $\alpha$-glycerophosphate, and also, when induced by G-6-P, by the UhpT system (Kahan et al. 1974). Both of these systems are positively regulated by CAMP, and cAMP levels can be lowered by mutations in the ptsI or cyaA genes (which will also affect catabolism of a variety of carbohydrates) (Alper and Ames 1978; Tsuruoka et al. 1978; Castañeda-García et al. 2009). For E. coli grown in the absence of G6-P, in which only the GlpT permease is active, the frequency of resistance (ascertained by fluctuation test) is $10^{-7}$, while in the presence of G-6-P the frequency is $10^{-8}$ (Nilsson et al. 2003). Most of the mutations seen in the Nils- 
son study were located in genes leading to a decrease in cAMP. Nilsson noted that despite this finding of high frequencies of resistance in vitro the rates of clinical resistance to fosfomycin in E. coli throughout Europe from 1999 to 2000 (Kahlmeter 2000) were uniformly low, in the range of $0.7 \%$ to $1.5 \%$, with no differences between countries with a long history of fosfomycin use and those not using it. As opposed to their in vitro findings, 13 resistant clinical isolates tested by Nilsson were found to have mutations almost exclusively in $g l p T$ and/or $u h p A$ or $u h p T$, with none found in the cAMP regulatory loci, perhaps indicating a selective disadvantage in vivo. Whereas mutants grew well in the absence of fosfomycin, it was found that growth rates of the three tested in vitro mutants and 12 of the 13 clinical isolates were severely reduced in growth rate in LB medium or urine in the presence of $\geq 8 \mathrm{mg} / \mathrm{L}$ of fosfomycin, which is significantly lower than the fosfomycin concentration in urine during treatment, normally greater than $128 \mathrm{mg} / \mathrm{L}$. Mathematical modeling indicated that the growth rate retardation seen should be enough to prevent resistant strains from establishment in the bladder (Nilsson et al. 2003). Slow growth and the absence of the cAMP-related mutations in vivo could explain the discrepancy between high-frequency mutational resistance to fosfomycin in vitro and rates of resistance seen in the clinic.

Interestingly, very few instances have been reported of mutations in the fosfomycin target gene, murA. A mutation in murA of E. coli was isolated after mutagenesis ( $\mathrm{Wu}$ and Venkateswaran 1974) and counterselection against transport mutants and two murA mutants of E. coli were reported among clinical isolates in a Japanese study (Takahata et al. 2010) It is likely that, in vitro, the frequency of transport mutants is so great relative to target mutants that they are not generally seen. The finding of murA mutants with clinical isolates of $E$. coli might reflect the low fitness of in vitro transport mutants.

Recent in vitro experiments (Couce et al. 2012) showed that overexpression of the murA gene by induction of a regulated promoter can lead to greatly increased MICs, to levels that would afford clinical resistance, while having relatively low effects on fitness (relative to mutations to fosfomycin resistance found in clinical isolates). However, this has not been noted yet in clinical isolates.

A study of a set of 441 Italian Gram-negative urinary isolates (Marchese et al. 2003) showed very high susceptibility to fosfomycin of E. coli isolates (99\%), 87.5\% susceptibility for Proteus species, whereas other species showed variable susceptibilities. Fosfomycinresistant mutants $(\geq 2000 \mathrm{mg} / \mathrm{L})$ of sensitive E. coli, K. pneumoniae, and P. aeruginosa were obtained through stepwise selection on agar containing fosfomycin, and the growth rates of these resistant mutants were compared to their parental strains. In contrast to the mutants tested by Nilsson et al., these all showed significantly reduced growth rates in a variety of conditions (in the absence of fosfomycin) as well as reduced adhesion to uroepithelial cells and to urinary catheters.

A study of fosfomycin resistance in Pseudomonas (Rodríguez-Rojas et al. 2010b) showed that a $g l p T$ null mutant was equal in virulence to its $g l p^{+}$parent in a mouse lung infection survival model, had equivalent biofilm-forming ability, and caused similar inflammation in histological studies. This and other data were interpreted by the authors to show that mutational resistance in $P$. aeruginosa may have no obvious fitness cost in vivo. It may well be that the site of infection contributes to the difference in apparent fitness seen between $P$. aeruginosa in the lung and E. coli in the bladder.

As there is interest in use of parenteral fosfomycin for treatment of indications in addition to uncomplicated UTI, recent hollow fiber pharmacokinetic/pharmacodynamic (PK/PD) studies were undertaken by the Ambrose group (VanScoy et al. 2015) to determine the PK/PD index for IV fosfomycin and to determine requirements for stasis, and 1- and 2-log reductions of colony-forming units. The results showed that, at least for the E. coli isolates studied, there was a large resistant subpopulation from the inception of infection. Thus, a new $\mathrm{PK} / \mathrm{PD}$ index was instituted, \% $>\mathrm{T}>\mathrm{RIC}$ - the percentage of the dosing interval in which the 
L.L. Silver

concentration was above the "resistance inhibitory concentration.” For stasis, 1- and 2-log reductions, $\% \mathrm{~T}>\mathrm{RIC}$ were $11.9 \%, 20.9 \%$, and $32.8 \%$, respectively. The authors note some caveats with this type of in vitro work: in vivo fitness of the mutants is unknown and longer treatment will be required to model clinical treatment duration.

In vitro pharmacodynamics of $\mathrm{Na}_{2}$-fosfomycin against clinical $64 \mathrm{P}$. aeruginosa isolates was studied by an Australian group (Walsh et al. 2015). MICs from 1 to $>512$ were seen with $61 \%$ of isolates susceptible (MIC $\leq 64 \mathrm{mg} / \mathrm{L}$ ), but all isolates tested had a resistant subpopulation as revealed by population analysis profiling. At low inocula $\left(\sim 10^{6} \mathrm{cfu} / \mathrm{mL}\right)$, there was moderate killing with regrowth by $24 \mathrm{~h}$ at most dosages. No killing was seen with high inocula $\left(\sim 10^{8} \mathrm{cfu} / \mathrm{mL}\right)$. This indicates that monotherapy of $P$. aeruginosa with fosfomycin may be problematic if this finding translates to in vivo conditions; furthermore, study of combination therapy is warranted.

\section{Fosfomycin-Modifying Enzymes}

Several fosfomycin-modifying enzymes have been found that inactivate the drug. The main enzymes described are three types of metalloenzymes, FosA, FosB, and FosX, and two kinases, FomA and FomB, as shown in Figure 3.

The metalloenzymes open the epoxide (oxirane ring) by the addition of various substrates as recently reviewed (Castañeda-García et al. 2013). FosA is a glutathione- $S$-transferase present on transposon TN2921 originally found on a plasmid in S. marcescens (Suárez and Mendoza 1991), using $\mathrm{Mn}^{2+}$ and $\mathrm{K}^{+}$as metal cofactors; other related glutathione transferase (FosA type) enzymes found to be plasmid-borne are

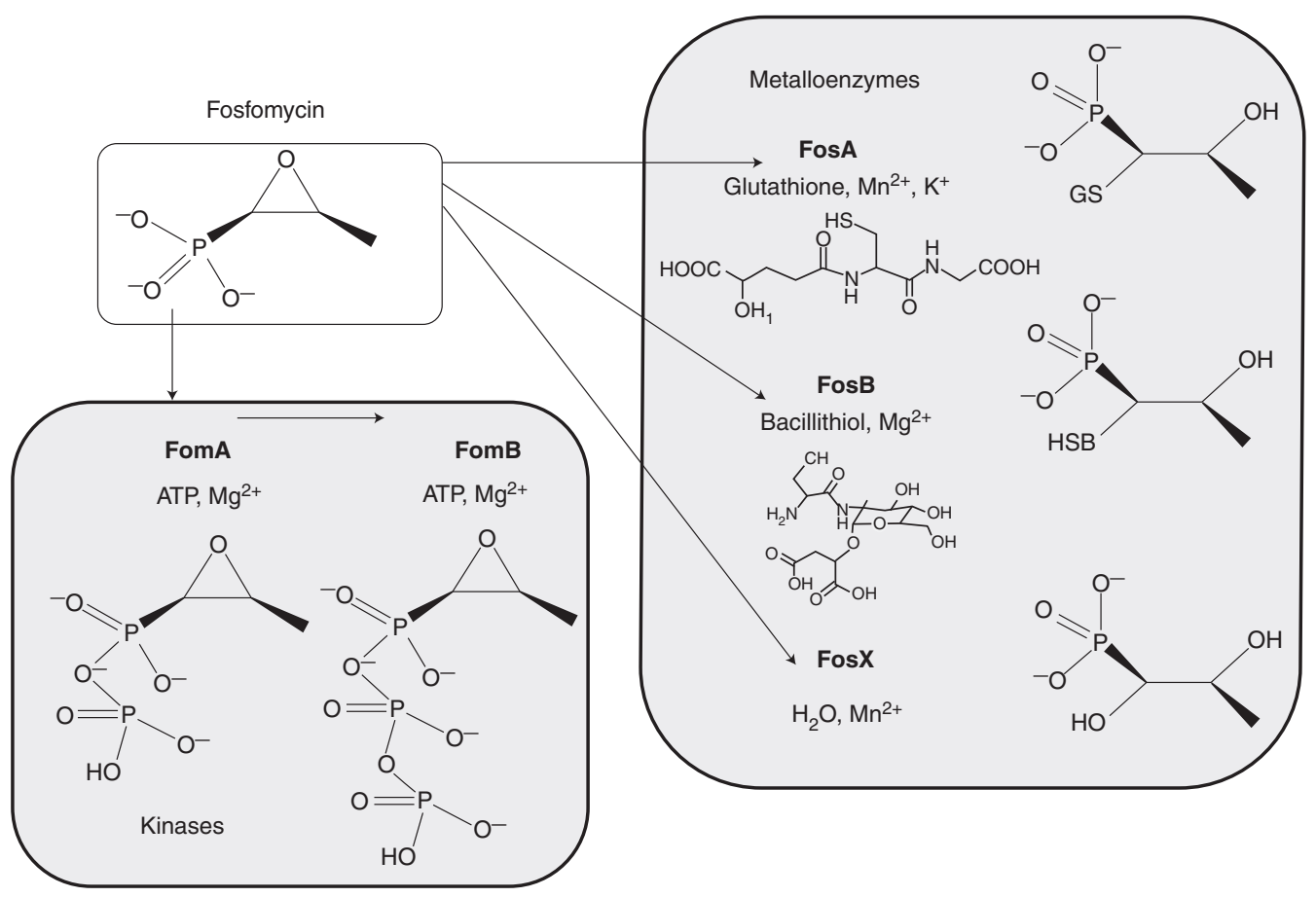

Figure 3. Fosfomycin-modifying enzymes leading to inactivation of fosfomycin and responsible for fosfomycin resistance. Enzyme names are in bold and are shown with their substrates, metal cofactors, and the end products of their modifying activity. (This figure is adapted from Castañeda-García et al. 2013 and is used under license from Creative Commons, creativecommons.org/licenses/by/3.0.) 
FosA3, FosA4, FosA5, and FosC2. A related FosA enzyme is found encoded in the chromosome of $P$. aeruginosa.

Recent studies in China ( $\mathrm{Li}$ et al. 2015) found that $7.8 \%$ of nonduplicate E. coli clinical isolates collected from 20 geographically dispersed hospitals from July 2009 to June 2010 were nonsusceptible to fosfomycin (MIC > $64 \mathrm{mg}$ per liter). Of these, $80 \%$ carried the fosA 3 gene; the fosA 3 gene of $42 \%$ of those isolates was transferable, presumably via a conjugative plasmid. With growing interest in the possible use of fosfomycin to combat resistant Gram-negative infections, a study was done to ascertain the prevalence of fosfomycin resistance in $278 \mathrm{~K}$. pneumoniae isolates carrying the K. pneumoniae carbapenemase (KPC) and in 80 extended-spectrum $\beta$-lactams (ESBLs) (non-KPC) K. pneumoniae from 12 hospitals in China (Jiang et al. 2015). Fosfomycin resistance was $60.8 \%$ in KPC producers, compared to $12.5 \%$ among ESBL producers. Ninety-four of the KPC isolates were found to carry fos $A 3$ genes and 92 of those appeared clonally related, with $71 \%$ belonging to a single clonal group. The distribution of fosfomycin resistance levels varied greatly among the different hospitals, but there was general correlation between levels of resistance and presence of fos 3 genes. Rather alarming was the finding that, in a representative isolate of the predominant clone, the fos $A 3$ and $b l a_{\mathrm{KPC}-2}$ genes were colocalized on a plasmid designated pFOS18. The $b l a_{\mathrm{KPC}-2}$ gene, located on a Tn3-Tn4401 structure, and fosA3, bracketed by IS26 sequences, are normally transmitted on plasmids. This data indicates that they can be linked on a single plasmid. The data from this recent study in China contrasts with earlier data from a U.S. study (Endimiani et al. 2010) in which fosfomycin susceptibility of $68 \mathrm{~K}$. pneumoniae KPC-possessing strains was measured and $93 \%$ were found susceptible. It is likely that the Chinese study represents a clonal outbreak that has not (yet) spread to the United States. It will be necessary to monitor the spread of such clones.

FosA3 was also found on CTX-M plasmids of E. coli in Japan, flanked there as well by IS26 elements (Sato et al. 2013). An unexpected in- crease in fosfomycin resistance of E. coli CTXM-15-carrying strains in Madrid was studied and found to be caused by two main clonal types (Oteo et al. 2009). In the larger clone, none of the normal mutational resistance alleles were found, whereas in the second clone of five strains, there was a small deletion in the uhpA gene and a single IS26 linked to bla $a_{\mathrm{CTX}-\mathrm{M}-15}$. There was no mention of genes associated with the IS26. Is it possible that these clones could contain fosfomycin-modifying enzymes, perhaps Fos3 associated with CTX-M as in the Japanese isolates (Sato et al. 2013)?

The regulation of expression of the fos $A$ gene that is resident in the genome of $P$. aeruginosa is not well studied, if at all. It would seem critically important to study the conditions under which fos $A$ is expressed in $P$. aeruginosa, to ascertain what its effect is on basal MICs and whether sufficient expression to yield a resistant phenotype can be achieved by mutation or induction. One reference (De Groote et al. 2011) notes that fosfomycin resistance in $P$. aeruginosa can be caused by (engineered) overexpression of the resident FosA because of an inserted promoter. Whether turn-on of FosA in P. aeruginosa is a possible source of mutational resistance is not known. However, the main mutational resistance in $P$. aeruginosa is clearly mutations in the GlpT system (Castañeda-García et al. 2009), because no spontaneous fosfomycin-resistant colonies grew on plates containing $\alpha$ glycerophosphate as a carbon source, and all 10 sequenced mutations in this study were missense mutations in $g l p T$.

FosB type enzymes, first discovered in S. epidermidis (Zilhao and Courvalin 1990), are found in low GC Gram-positive bacteria such as B. subtilis, B. anthracis, S. epidermidis, and $S$. aureus, species that do not make glutathione but use bacillithiol (shown in Fig. 3) as a substitute thiol donor. These bacillithiol- $S$ transferases use $\mathrm{Mg}^{2+}$ as a cofactor. The FosB of $S$. aureus is chromosomally located and is responsible for the innate level of fosfomycin activity - as deletion of either the fos $B$ gene or the bacillithiol synthetic machinery greatly increases the sensitivity to fosfomycin (Thompson et al. 2014). 
FosX is related to the FosA and FosB enzymes, but it is an $\mathrm{Mn}^{2+}$-dependent epoxide hydrolase, using water to break the ring. FosX enzymes are found in Listeria monocytogenes, Clostridium botulinum, and Brucella melitensis (Castañeda-García et al. 2013).

FomA and FomB are kinases from S. wedmorensis, a species that produces fosfomycin (Kuzuyama et al. 1996), that sequentially add phosphates to the phosphonate moiety of fosfomycin from ATP with $\mathrm{Mg}^{2+}$ as a cofactor. Presumably, these enzymes act to provide autoresistance to the producers. Another such kinase, originally called FosC and found in the fosfomycin producer $P$. syringae, is actually an ortholog of FomA (Kim et al. 2012).

\section{CLINICAL CONSIDERATIONS ON RESISTANCE}

As fosfomycin has low toxicity and allergenicity, a broad spectrum of activity, including MDR organisms, good pharmacokinetics, and is available in parenteral as well as oral formulations, its use in non-UTI indications for hardto-treat infections has become attractive. But there is not a large body of data on results of controlled trials in other indications. The Falagas group has published a large number of meta-analyses of small studies of fosfomycin use in various settings. Initial data supported use of fosfomycin for treatment of UTIs caused by MDR Enterobacteriaceae, including those carrying ESBLs (Falagas et al. 2010a). Resistance data from studies after 2010 (Falagas et al. 2016) was mostly on pathogens from urine samples and showed that E. coli susceptibility ranged from $82 \%$ to $100 \%$ and $K$. pneumoniae from $15 \%$ to $100 \%$. There was less data on other Enterobacteriaceae, but fosfomycin was active against $72 \%$ to $97.5 \%$. Fosfomycin was $90.5 \%$ to $100 \%$ active against MDR Enterobacteriaceae. One study showed $80.6 \%$ susceptibility to fosfomycin of carbapenem-resistant P. aeruginosa strains. Carbapenem-resistant Acinetobacter baumannii was resistant. No major differences in resistance were seen between data published before 2010 and that published after for Gram-negatives and S. aureus.
The main question is whether resistance to fosfomycin will compromise its use in nonUTI indications. There is no adequate answer as yet. It is clear that the single-dose oral treatment of cystitis with fosfomycin trometamol/ tromethamine is highly effective and equivalent to other therapies with low evolution of resistance seen (Falagas et al. 2010b). Most of these infections are caused by E. coli, and it may be that the high urinary levels of fosfomycin attainable and the lowered fitness of mutants with impaired fosfomycin uptake (as discussed above) contribute to the efficacy of fosfomycin in this setting.

\section{Combinations}

Checkerboard assays, kill curves, and in vitro models have been used to predict the efficacy and potential synergy of combinations of fosfomycin. Whereas monotherapy against E. coli and E. faecalis cystitis has been successful over the years, there is concern that treatment of other pathogens at other sites where local drug concentrations will likely be lower than in urine may lead to resistance selection. Thus, combination therapy is being evaluated.

Combinations with underused cell-wall inhibitors were tested against E. coli and K. pneumoniae (Hickman et al. 2013), including MDR strains, leading to the finding that a fosfomycin/aztreonam and a fosfomycin/aztreonam/ mecillinam combination were effective in reducing the population in a variety of models, including a new in vitro kinetic model.

An examination of resistance selection by fosfomycin in combination with a number of drugs in both wild-type and a mutator strain (Rodríguez-Rojas et al. 2010a) gave the interesting result that, in wild-type, combinations with tobramycin, amikacin, meropenem, ceftazidime, ciprofloxacin, and colistin gave frequencies below the limit of detection $\left(<1 \times 10^{-10}\right)$, but the combination of fosfomycin and imipenem had a frequency of $1.1 \times 10^{-9}$, higher than the product of the individual frequencies (3.5 $\left.\times 10^{-13}\right)$. For the mutator strain, in which frequencies for the single drugs were $\sim 100$ fold higher than in wild-type, all combinations 
yielded frequencies below the limit of detection except for fosfomycin plus imipenem or ceftazidime, which were $1.1 \times 10^{-7}$ and $1 \times 10^{-8}$ respectively. There was, apparently, some sort of antagonistic effect occurring.

A recent publication (Walsh et al. 2016) reported testing combinations of fosfomycin with several drugs in killing $P$. aeruginosa. Against fosfomycin-susceptible isolates, fosfomycin monotherapy led to efficient killing but rapid regrowth. Combination of fosfomycin with polymyxin B or tobramycin against these susceptible isolates and combination of ciprofloxacin plus fosfomycin against fosfomycin-resistant isolates led to increased killing (versus monotherapy), but did not prevent regrowth of resistant mutants. Certainly, repeat dosing should be tried to ascertain whether rebound could be prevented with further treatment, but this study is sobering.

\section{CONCLUSIONS}

Fosfomycin is an old antibiotic, but it has proven useful. In this age of increasing antibiotic resistance, fosfomycin is being reconsidered for use against MDR pathogens within its spectrum of action. But, if it is true that fosfomycin has proven effective for uncomplicated UTIs because of fitness cost of mutants and high urinary drug concentrations, then these are variables that must be addressed to move on to indications involving other body sites. The use of combinations may help to keep resistance at bay, but that will likely rely on matched pharmacokinetics. Furthermore, data from various tests of combinations against $P$. aeruginosa show the need to choose combinations carefully. Dosing of drug at "resistance-inhibitory" or "mutant-prevention" concentrations may be a more realistic approach. Whereas the frequencies of fosfomycin resistance seen over time have not changed very much, that is likely because of the fact that most of the present resistance seen is a result of mutations in the pathogen. The reports on plasmid-borne fosfomycin resistance forewarn of increased levels of resistance, likely through spread of problematic clones in which resistance to fosfomycin is linked to resistance to other drugs, especially $\beta$-lactams. The Chinese report of FosA 3 and KPC-2 on a single plasmid and its likely clonal spread (Jiang et al. 2015) are very worrying. Whereas drugs to treat the growing threat of MDR bacteria are sorely needed, and fosfomycin has some attractive properties that could favor its use for treatment of certain life-threatening infections, to retain its productive use as a single-dose oral therapy for cystitis, stewardship and monitoring the spread of plasmid-borne resistance will be needed.

\section{REFERENCES}

Alper MD, Ames BN. 1978. Transport of antibiotics and metabolite analogs by systems under cyclic AMP control: Positive selection of Salmonella typhimurium cya and crp mutants. J Bacteriol 133: 149-157.

Blake KL, O’Neill AJ, Mengin-Lecreulx D, Henderson PJ, Bostock JM, Dunsmore CJ, Simmons KJ, Fishwick CW, Leeds JA, Chopra I. 2009. The nature of Staphylococcus aureus MurA and MurZ and approaches for detection of peptidoglycan biosynthesis inhibitors. Mol Microbiol 72: 335-343.

Brown ED, Marquardt JL, Lee JP, Walsh CT, Anderson KS. 1994. Detection and characterization of a phospholactoyl-enzyme adduct in the reaction catalyzed by UDP$\mathrm{N}$-acetylglucosamine enolpyruvoyl transferase, MurZ. Biochemistry 33: 10638-10645.

Castañeda-García A, Rodríguez-Rojas A, Guelfo JR, Blázquez J. 2009. The glycerol-3-phosphate permease GlpT is the only fosfomycin transporter in Pseudomonas aeruginosa. J Bacteriol 191: 6968-6974.

Castañeda-García A, Blázquez J, Rodríguez-Rojas A. 2013. Molecular mechanisms and clinical impact of acquired and intrinsic fosfomycin resistance. Antibiotics 2: 217236.

Couce A, Briales A, Rodríguez-Rojas A, Costas C, Pascual A, Blázquez J. 2012. Genomewide overexpression screen for fosfomycin resistance in Escherichia coli: MurA confers clinical resistance at low fitness cost. Antimicrob Agents Chemother 56: 2767-2769.

De Groote VN, Fauvart M, Kint CI, Verstraeten N, Jans A, Cornelis P, Michiels J. 2011. Pseudomonas aeruginosa fosfomycin resistance mechanisms affect non-inherited fluoroquinolone tolerance. J Med Microbiol 60: 329-336.

De Smet KAL, Kempsell KE, Gallagher A, Duncan K, Young DB. 1999. Alteration of a single amino acid residue reverses fosfomycin resistance of recombinant MurA from Mycobacterium tuberculosis. Microbiology 145: 31773184.

Du W, Brown JR, Sylvester DR, Huang J, Chalker AF, So CY, Holmes DJ, Payne DJ, Wallis NG. 2000. Two active forms of UDP- $N$-acetylglucosamine enolpyruvyl transferase in Gram-positive bacteria. J Bacteriol 182: 4146-4152.

Endimiani A, Patel G, Hujer KM, Swaminathan M, Perez F, Rice LB, Jacobs MR, Bonomo RA. 2010. In vitro activity 
of fosfomycin against $b l a_{\mathrm{KPC}}$-containing Klebsiella pneumoniae isolates, including those nonsusceptible to tigecycline and/or colistin. Antimicrob Agents Chemother 54: 526-529.

Eschenburg S, Priestman M, Schonbrunn E. 2005. Evidence that the fosfomycin target Cys115 in UDP- $N$-acetylglucosamine enolpyruvyl transferase (MurA) is essential for product release. J Biol Chem 280: 3757-3763.

European Committee on Antimicrobial Susceptibility Testing. 2016. Breakpoint tables for interpretation of MICs and zone diameters. Version 6. www.eucast.org/ clinical_breakpoints.

Falagas ME, Giannopoulou KP, Kokolakis GN, Rafailidis PI 2008. Fosfomycin: Use beyond urinary tract and gastrointestinal infections. Clin Inf Dis 46: 1069-1077.

Falagas ME, Kastoris AC, Karageorgopoulos DE, Rafailidis PI. 2009. Fosfomycin for the treatment of infections caused by multidrug-resistant non-fermenting Gramnegative bacilli: A systematic review of microbiological, animal and clinical studies. Int J Antimicrob Agents 34: $111-120$.

Falagas ME, Kastoris AC, Kapaskelis AM, Karageorgopoulos DE. 2010a. Fosfomycin for the treatment of multidrugresistant, including extended-spectrum $\beta$-lactamase producing, Enterobacteriaceae infections: A systematic review. Lancet Inf Dis 10: 43-50.

Falagas ME, Vouloumanou EK, Togias AG, Karadima M, Kapaskelis AM, Rafailidis PI, Athanasiou S. 2010b. Fosfomycin versus other antibiotics for the treatment of cystitis: A meta-analysis of randomized controlled trials. J Antimicrob Chemother 65: 1862-1877.

Falagas ME, Vouloumanou EK, Samonis G, Vardakas KZ. 2016. Fosfomycin. Clin Microbiol Rev 29: 321-347.

Gadebusch HH, Stapley EO, Zimmerman SB. 1992. The discovery of cell wall active antibacterial antibiotics. Crit Rev Biotechnol 12: 225-243.

Hendlin D, Stapley EO, Jackson M, Wallick H, Miller AK, Wolf FJ, Miller TW, Chaiet L, Kahan FM, Foltz EL, et al. 1969. Phosphonomycin, a new antibiotic produced by strains of Streptomyces. Science 166: 122-123.

Hickman RA, Hughes D, Cars T, Malmberg C, Cars O. 2013. Cell-wall-inhibiting antibiotic combinations with activity against multidrug-resistant Klebsiella pneumoniae and Escherichia coli. Clin Microbiol Inf 20: O267-O273.

Jiang Y, Shen P, Wei Z, Liu L, He F, Shi K, Wang Y, Wang H, Yu Y. 2015. Dissemination of a clone carrying a fosA3-harbouring plasmid mediates high fosfomycin resistance rate of KPC-producing Klebsiella pneumoniae in China. Int J Antimicrob Agents 45: 66-70.

Kahan FM, Kahan JS, Cassidy PJ, Kropp H. 1974. The mechanism of action of fosfomycin (phosphonomycin). Ann NYAcad Sci 235: 364-386.

Kahlmeter G. 2000. The ECO•SENS Project: A prospective, multinational, multicentre epidemiological survey of the prevalence and antimicrobial susceptibility of urinary tract pathogens-Interim report. J Antimicrob Chemother 46: 15-22.

Karageorgopoulos DE, Wang R, Yu XH, Falagas ME. 2012. Fosfomycin: Evaluation of the published evidence on the emergence of antimicrobial resistance in Gram-negative pathogens. J Antimicrob Chemother 67: 255-268.
Katayama N, Tsubotani S, Nozaki Y, Harada S, Ono H. 1990. Fosfadecin and fosfocytocin, new nucleotide antibiotics produced by bacteria. J Antibiot (Tokyo) 43: 238-246.

Kedar GC, Brown-Driver V, Reyes DR, Hilgers MT, Stidham MA, Shaw KJ, Finn J, Haselbeck RJ. 2008. Comparison of the essential cellular functions of the two murA genes of Bacillus anthracis. Antimicrob Agents Chemother 52: 2009-2013.

Kim DH, Lees WJ, Kempsell KE, Lane WS, Duncan K, Walsh CT. 1996. Characterization of a Cys115 to Asp substitution in the Escherichia coli cell wall biosynthetic enzyme UDP-GlcNAc enolpyruvyl transferase (MurA) that confers resistance to inactivation by the antibiotic fosfomycin. Biochemistry 35: 4923-4928.

Kim SY, Ju K-S, Metcalf WW, Evans BS, Kuzuyama T, van der Donk WA. 2012. Different biosynthetic pathways to fosfomycin in Pseudomonas syringae and Streptomyces species. Antimicrob Agents Chemother 56: 4175-4183.

Kobayashi K, Ehrlich SD, Albertini A, Amati G, Andersen KK, Arnaud M, Asai K, Ashikaga S, Aymerich S, Bessieres P. 2003. Essential Bacillus subtilis genes. Proc Natl Acad Sci 100: $4678-4683$.

Kock H, Gerth U, Hecker M. 2004. MurAA, catalysing the first committed step in peptidoglycan biosynthesis, is a target of Clp-dependent proteolysis in Bacillus subtilis. Mol Microbiol 51: 1087-1102.

Kuzuyama T, Kobayashi S, O’Hara K, Hidaka T, Seto H. 1996. Fosfomycin monophosphate and fosfomycin diphosphate, two inactivated fosfomycin derivatives formed by gene products of fom $A$ and fomB from a fosfomycin producing organism Streptomyces wedmorensis. J Antibiot (Tokyo) 49: 502-504.

Lemieux MJ, Huang Y, Wang DN. 2004. Glycerol-3-phosphate transporter of Escherichia coli: Structure, function and regulation. Res Microbiol 155: 623-629.

Li Y, Zheng B, Li Y, Zhu S, Xue F, Liu J. 2015. Antimicrobial susceptibility and molecular mechanisms of fosfomycin resistance in clinical Escherichia coli isolates in mainland China. PLoS ONE 10: e0135269.

Marchese A, Gualco L, Debbia EA, Schito GC, Schito AM. 2003. In vitro activity of fosfomycin against Gram-negative urinary pathogens and the biological cost of fosfomycin resistance. Int J Antimicrob Agents 22: 53-59.

Marquardt JL, Brown ED, Lane WS, Haley TM, Ichikawa Y, Wong CH, Walsh CT. 1994. Kinetics, stoichiometry, and Identification of the reactive thiolate in the inactivation of UDP-GlcNAc enolpyruvoyl transferase by the antibiotic fosfomycin. Biochemistry 33: 10646-10651.

Nikolaidis I, Favini-Stabile S, Dessen A. 2014. Resistance to antibiotics targeted to the bacterial cell wall. Protein Sci 23: $243-259$.

Nilsson AI, Berg OG, Aspevall O, Kahlmeter G, Andersson DI. 2003. Biological costs and mechanisms of fosfomycin resistance in Escherichia coli. Antimicrob Agents Chemother 47: 2850-2858.

Oteo J, Orden B, Bautista V, Cuevas O, Arroyo M, MartinezRuiz R, Perez-Vazquez M, Alcaraz M, Garcia-Cobos S, Campos J. 2009. CTX-M-15-producing urinary Escherichia coli O25b-ST131-phylogroup B2 has acquired resistance to fosfomycin. J Antimicrob Chemother 64: 712717. 
Fosfomycin: Mechanism and Resistance

Performance Standards for Antimicrobial Susceptibility Testing. 2012. 22nd informational supplement M100S22. Clinical and Laboratory Standards Institute, Wayne, PA.

Rodríguez-Rojas A, Couce A, Blázquez J. 2010a. Frequency of spontaneous resistance to fosfomycin combined with different antibiotics in Pseudomonas aeruginosa. Antimicrob Agents Chemother 54: 4948-4949.

Rodríguez-Rojas A, Maciá MD, Couce A, Gómez C, Castañeda-García A, Oliver A, Blázquez J. 2010b. Assessing the emergence of resistance: The absence of biological cost in vivo may compromise fosfomycin treatments for P. aeruginosa infections. PLOS ONE 5: e10193.

Sato N, Kawamura K, Nakane K, Wachino JI, Arakawa Y 2013. First detection of fosfomycin resistance gene fosA3 in CTX-M-producing Escherichia coli isolates from healthy individuals in Japan. Microb Drug Res 19: 477-482.

Shoji J, Kato T, Hinoo H, Hattori T, Hirooka K, Matsumoto K, Tanimoto T, Kondo E. 1986. Production of fosfomycin (phosphonomycin) by Pseudomonas syringae. J Antibiot (Tokyo) 39: 1011-1012.

Skarzynski T, Mistry A, Wonacott A, Hutchinson SE, Kelly VA, Duncan K. 1996. Structure of UDP- $N$-acetylglucosamine enolpyruvyl transferase, an enzyme essential for the synthesis of bacterial peptidoglycan, complexed with substrate UDP- $N$-acetylglucosamine and the drug fosfomycin. Structure 4: 1465-1474.

Suárez JE, Mendoza MC. 1991. Plasmid-encoded fosfomycin resistance. Antimicrob Agents Chemother 35: 791795.

Takahata S, Ida T, Hiraishi T, Sakakibara S, Maebashi K, Terada S, Muratani T, Matsumoto T, Nakahama C, Tomono K. 2010. Molecular mechanisms of fosfomycin resistance in clinical isolates of Escherichia coli. Int J Antimicrob Agents 35: 333-337.

Thompson MK, Keithly ME, Goodman MC, Hammer ND, Cook PD, Jagessar KL, Harp J, Skaar EP, Armstrong RN 2014. Structure and function of the genomically encoded fosfomycin resistance enzyme, FosB, from Staphylococcus aureus. Biochemistry 53: 755-765.

Tsuruoka T, Miyata A, Yamada Y. 1978. Two kinds of mutants defective in multiple carbohydrate utilization isolated from in vitro fosfomycin-resistant strains of Escherichia coli K-12. J Antibiot (Tokyo) 31: 192-201.
VanScoy BD, McCauley J, Ellis-Grosse EJ, Okusanya OO, Bhavnani SM, Forrest A, Ambrose PG. 2015. Exploration of the pharmacokinetic-pharmacodynamic relationships for fosfomycin efficacy using an in vitro infection model. Antimicrob Agents Chemother 59: 7170-7177.

Walsh CC, McIntosh MP, Peleg AY, Kirkpatrick CM, Bergen PJ. 2015. In vitro pharmacodynamics of fosfomycin against clinical isolates of Pseudomonas aeruginosa. J Antimicrob Chemother 70: 3042-3050.

Walsh CC, Landersdorfer CB, McIntosh MP, Peleg AY, Hirsch EB, Kirkpatrick CM, Bergen PJ. 2016. Clinically relevant concentrations of fosfomycin combined with polymyxin B, tobramycin or ciprofloxacin enhance bacterial killing of Pseudomonas aeruginosa, but do not suppress the emergence of fosfomycin resistance. J Antimicrob Chemother doi: 10.1093/jac/dkw115.

Wanke C, Amrhein N. 1993. Evidence that the reaction of the UDP- $N$-acetylglucosamine 1-carboxyvinyltransferase proceeds through the $O$-phosphothioketal of pyruvic acid bound to Cys115 of the enzyme. Eur J Biochem 218: 861-870.

Winkler HH. 1973. Distribution of an inducible hexosephosphate transport system among various bacteria. $J$ Bacteriol 116: 1079-1081.

Wu HC, Venkateswaran PS. 1974. Fosfomycin-resistant mutant of Escherichia coli. Ann NY Acad Sci 235: 587-592.

Zavante Therapeutics. 2016. Zavante initiates the ZEUS study for ZTI-01 for the treatment of complicated urinary tract infections, Zavante Therapeutics, Inc., www.zavante.com/news/zavante-initiates-the-zeus-studyfor-zti-01-for-the-treatment-of-complicated-urinary-tractinfections.

Zhu JY, Yang Y, Han H, Betzi S, Olesen SH, Marsilio F, Schönbrunn E. 2012. Functional consequence of covalent reaction of phosphoenolpyruvate with UDP-N-acetylglucosamine 1-carboxyvinyltransferase (MurA). J Biol Chem 287: 12657-12667.

Zilhao R, Courvalin P. 1990. Nucleotide sequence of the fos $B$ gene conferring fosfomycin resistance in Staphylococcus epidermidis. FEMS Microbiol Lett 68: 267-272.

Zimmerman S, Stapley E, Wallick H, Baldwin R. 1969. Phosphonomycin. IV: Susceptibility testing method and survey. Antimicrob Agents Chemother (Bethesda) 9:303-309. 


\title{
$\&_{\mathrm{CSH}}^{\infty} \&$ Cold Spring Harbor

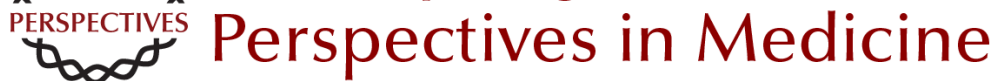

\section{Fosfomycin: Mechanism and Resistance}

\author{
Lynn L. Silver
}

Cold Spring Harb Perspect Med 2017; doi: 10.1101/cshperspect.a025262 originally published online January 6, 2017

\section{Subject Collection Antibiotics and Antibiotic Resistance}

Fosfomycin: Mechanism and Resistance Lynn L. Silver

Pleuromutilins: Potent Drugs for Resistant Bugs

--Mode of Action and Resistance

Susanne Paukner and Rosemarie Riedl

Appropriate Targets for Antibacterial Drugs Lynn L. Silver

Lincosamides, Streptogramins, Phenicols, and Pleuromutilins: Mode of Action and Mechanisms of Resistance

Stefan Schwarz, Jianzhong Shen, Kristina Kadlec, et al.

Resistance to Macrolide Antibiotics in Public Health Pathogens

Corey Fyfe, Trudy H. Grossman, Kathy Kerstein, et al.

Bacterial Protein Synthesis as a Target for

Antibiotic Inhibition

Stefan Arenz and Daniel N. Wilson

Antibacterial Antifolates: From Development through Resistance to the Next Generation

Alexavier Estrada, Dennis L. Wright and Amy C. Anderson

Antibacterial Drug Discovery Targeting the Lipopolysaccharide Biosynthetic Enzyme LpxC Alice L. Erwin
The Whys and Wherefores of Antibiotic

Resistance

Cameron R. Strachan and Julian Davies

$\beta$-Lactamases: A Focus on Current Challenges Robert A. Bonomo

Approved Glycopeptide Antibacterial Drugs: Mechanism of Action and Resistance Daina Zeng, Dmitri Debabov, Theresa L. Hartsell, et al.

Mechanism of Action and Resistance to Daptomycin in Staphylococcus aureus and Enterococci William R. Miller, Arnold S. Bayer and Cesar A. Arias

Polymyxin: Alternative Mechanisms of Action and Resistance

Michael J. Trimble, Patrik Mlynárcik, Milan Kolár, et al.

Topoisomerase Inhibitors: Fluoroquinolone

Mechanisms of Action and Resistance David C. Hooper and George A. Jacoby

$\beta$-Lactams and $\beta$-Lactamase Inhibitors: An Overview Karen Bush and Patricia A. Bradford

Rifamycins, Alone and in Combination David M. Rothstein

For additional articles in this collection, see http://perspectivesinmedicine.cshlp.org/cgi/collection/ 